\title{
Anchors away: contribution of a glycolipid anchor to bacterial invasion of host cells
}

\author{
Miriam J. Baron ${ }^{1}$ and Dennis L. Kasper ${ }^{1,2}$
}

${ }^{1}$ The Channing Laboratory and the Division of Infectious Diseases, Department of Medicine, Brigham and Women's Hospital, Boston, Massachusetts, USA. ${ }^{2}$ Department of Microbiology and Molecular Genetics, Harvard Medical School, Boston, Massachusetts, USA.

\begin{abstract}
Group B Streptococcus (GBS) is an important cause of infections, including meningitis. The molecular events underlying its pathogenesis are poorly understood. A study in this issue of the JCI reports that the GBS invasion-associated gene (iagA) contributes to meningeal infection and virulence by facilitating invasion of the cells that compose the blood-brain barrier and of other host cells (see the related article beginning on page 2499). The mechanism involved most likely relates to the gene product's role in synthesis of a glycolipid anchor for a bacterial cell-surface entity that interacts directly with host cells.
\end{abstract}

Streptococcus agalactiae (known as group B Streptococcus [GBS]), identified by the presence of a cell wall-associated antigen called the group B polysaccharide, is a frequent colonizer of the human gastrointestinal and gynecological tracts and an important cause of infection in pregnant/peripartum women, neonates, and nonpregnant adults with underlying conditions. Neonatal GBS disease - typically pneumonia, sepsis, and/or meningitis - contributes significantly to infant morbidity and mortality.

\section{Molecular mechanisms of GBS pathogenesis}

The pathogenic process can be viewed as a series of hurdles that the organism must overcome to invade a host. To cause disease in the newborn, for example, GBS must come into contact with the host, traverse the epithelial barrier (either by passing through a cell from apical to basilar surface or by squeezing through the junctions between epithelial cells, as has been described for group A Streptococcus; ref. 1), and enter the bloodstream.

The tools used to accomplish these tasks include products that bacteria may

Nonstandard abbreviations used: BBB, bloodbrain barrier; BMEC, brain microvascular endothelial cell; CPS, capsular polysaccharide; DGlcDAG, [Glucose $(\beta 1-6)$ Glucose $(\beta 1-3)$ (gentiobiosyl)diacylglycerol]; GBS, group B Streptococcus; iagA, invasion-associated gene; LTA, lipoteichoic acid.

Conflict of interest: The authors have declared that no conflict of interest exists.

Citation for this article: J. Clin. Invest. 115:2325-2327 (2005). doi:10.1172/JCI26285. express on the surface and/or secrete into the surrounding environment. In many cases, especially when a particular step in pathogenesis is vital to organism survival, bacteria are equipped with multiple apparatuses with seemingly overlapping function. For example, several GBS surface components interact with epithelial cells (Figure 1), including the capsular polysaccharide (CPS), whose structure determines serotype; the CPS impairs internalization by host epithelial cells but does not influence adhesion (2). Lipoteichoic acid (LTA) mediates adherence to adult and neonatal epithelial cells (3). Bacterial proteins also play a role: adhesion of GBS to epithelial cells decreases by up to $75 \%$ after treatment of bacteria with proteases, which degrade bacterial proteins $(2,4)$. Specifically, the GBS proteins $\alpha \mathrm{C}(5,6)$, $\beta$-hemolysin/cytolysin (7), Spb1 (8), FbsA (9), FbsB (10), and SCPB/C5a peptidase (11) contribute to epithelial cell adhesion and internalization of GBS.

At each step during the host invasion process, GBS may encounter innate or adaptive immune defense mechanisms. Successful bacterial pathogens have developed means for coping with or avoiding particular defense mechanisms, employing surface-anchored or secreted components. For example, invasive pathogens frequently have polysaccharide capsules that allow them - once inside the bloodstream - to withstand the attack of the complement system and phagocytic cells. In the case of GBS, CPS has terminal sialic acid residues on its structural repeating units that impair complement deposition, which is required for phagocytosis $(12,13)$.

To cause meningitis, GBS must reach the bloodstream, evade host defenses, and enter the CNS - a site protected by the blood-brain barrier (BBB). The BBB consists of a single layer of specialized, non-pinocytosing brain microvascular endothelial cells (BMECs) joined by continuous tight junctions (14). The molecular-level interactions of GBS with the BBB remain poorly understood.

\section{Mutation of invasion-associated gene results in a hypoinvasive, hypovirulent GBS phenotype}

In this issue of the JCI, Doran et al. (15) report that $\mathrm{BBB}$ invasion depends upon a GBS gene responsible for proper bacterial cell-surface anchoring of LTA. Specifically, allelic replacement of invasion-associated gene (iagA) in the GBS chromosome yielded a strain with a 4-fold lower rate of BMEC invasion than the wild-type parent strain. Single-gene complementation restored invasion activity to wild-type levels, which suggests that mutations in other sites or polar effects on adjacent genes were not contributing to the findings. The mutation did not affect cellular adhesion or intracellular survival of organisms, and the invasion effects were independent of CPS. In fact, through studies of acapsular mutants, the investigators have shown that CPS is not essential for BMEC invasion by GBS, which supports previously reported findings for pneumococci (16). Following i.v. GBS challenge in a mouse model of meningitis, iagA mutant strain survival in the bloodstream was reportedly comparable to that of wild-type GBS, but virulence was attenuated, as indicated by lower mortality rates ( $20 \%$ vs. $90 \%$ ). In line with the mortality data, the mutant strain penetrated into the CNS less effectively than the wild-type strain.

While these data (15) clearly indicate that the iagA gene product is important for BMEC invasion and subsequent men- 


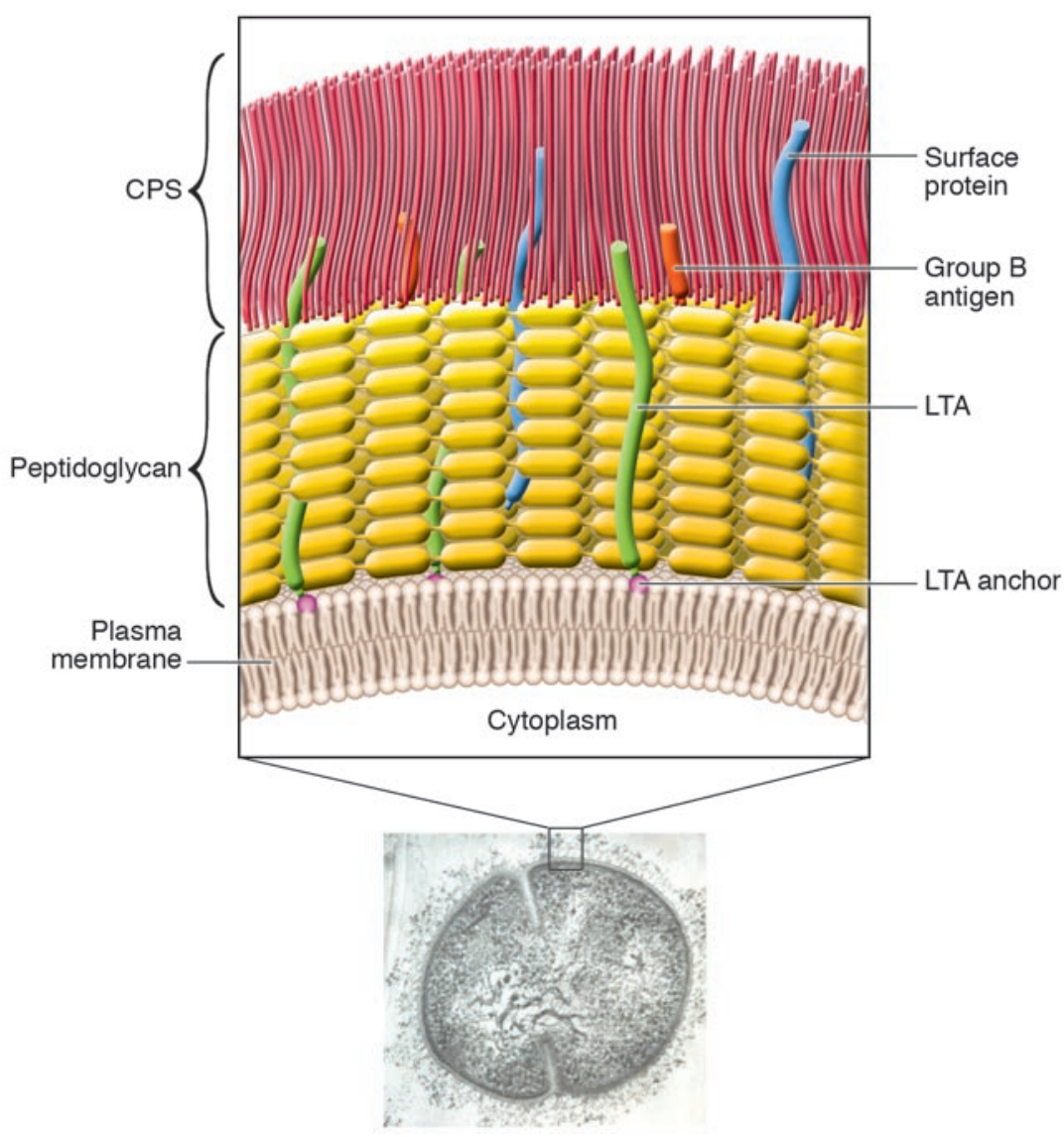

ingeal disease, several pieces of evidence suggest that the effects of this product are not specific to interactions with the BBB. First, in addition to diminished BMEC invasion, the iagA mutant strain exhibited hypoinvasiveness for lung and chorion epithelial cells. These data suggest that, rather than conferring a selective defect in CNS invasiveness, the iagA gene mutation leads to more global impairment of cell invasion. Second, the shared homology of the iagA gene product with gene products in other organisms (such as Enterococcus faecalis, Streptococcus mutans, and Acholeplasma laidlawii) that do not commonly cause meningitis reinforces the suggestion that this gene does not confer specifically the ability for bacteria to penetrate the BBB. Finally, although all GBS serotypes have the iagA gene, type III strains are responsible for the majority of neonatal meningitis cases (as opposed to only about $25 \%$ of cases of invasive neonatal disease overall) (17). The implication is that other mechanisms must contribute more specifically to human meningeal invasion. In summary, the iag $A$ gene product is clearly necessary for epithelial cell and BMEC invasion, but it is not the "meningitis factor."

\section{A role for LTA in iagA's contribution to GBS pathogenesis?}

Of particular interest is the question of how the gene product might facilitate invasion. The predicted gene product shares homology with the 1,2-diacylglycerol-3-glucose (1-2)-glucosyltransferase from $A$. laidlawii that synthesizes the glycolipid [Glucose $(\beta 1-6)$ Glucose $(\beta 1-3)$ (gen tiobiosyl)diacylglycerol] (DGlcDAG) (18). DGlcDAG acts as an anchor for LTA in several Gram-positive bacteria (19). Analysis of the glycolipid content of the GBS iag $A$ mutant by thin-layer chromatography supports the hypothesis that the iagA gene product acts as a DGlcDAG synthase (15). However, the contribution of LTA to the mutant phenotype remains unclear. Using a monoclonal antibody against LTA in an ELISA with intact organisms and culture supernatants, the authors found that LTA levels were higher on the surface of the mutant strain and in its culture supernatant than on the wild-type strain and in its supernatant (15). In an acapsu-

\section{Figure 1}

Cell wall structure of GBS. Electron micrograph image of a type III GBS organism labeled with antiserum raised to whole organism; the inset shows detailed representation of important surface structures. The GBS cytoplasm is bounded by an inner cell membrane. Surrounding this membrane is a peptidoglycan layer that anchors the negatively-charged CPS and group B antigen polysaccharide. Extending from the cell membrane are lipoproteins and glycolipids, including an anchor for LTA; LTA is a structure composed of a repeating carbohydrate phosphate polymer. Most surface proteins attach to the peptidoglycan. CPS, LTA, and cell-surface proteins contribute to the organism's ability to adhere to and invade host cells and evade host immune defenses in the course of pathogenesis. Data reported by Doran et al. (15) in this issue of the $\mathrm{JCl}$ reveal that an enzyme involved in the synthesis of a glycolipid anchor is required for normal GBS invasion of host cells in vitro and for GBS virulence in vivo. lar strain, however, iagA gene disruption was associated with lower organism surface LTA levels than were found on the surface of an acapsular strain with intact iagA function. The investigators conclude from these puzzling data that iagA gene disruption results in release of unanchored LTA from the cell wall but that this material becomes "trapped" by the CPS and remains detectable on the bacterial surface unless the organism is acapsular, in which case the LTA is released into the supernatant. This concept is difficult to understand because CPS is negatively charged (20), just as LTA presumably is (being an acid by definition, although its structure has not been elucidated); binding of one negatively charged molecule to another is unlikely. These data raise the question of whether the iagA gene product plays a role in the bacterium unrelated to anchoring LTA to the cell membrane, perhaps attaching another GBS structural component to the cell membrane.

In exploring the mechanism of the $i a g A$ mutant strain phenotype, the authors assess the roles of TLR2 and soluble LTA released from GBS (15). In other organ- 
isms, LTA interacts with TLR2, contributing to stimulation of cytokine-mediated host inflammatory responses that ultimately result in septic shock, circulatory collapse, and multiorgan failure in animal models $(21,22)$. Several lines of evidence in this study strongly suggest that the effects of iagA gene disruption in GBS are TLR2 independent (15). In addition, BMEC invasion by the wild-type strain was inhibited by the mutant strain supernatant but was enhanced by the wild-type strain supernatant. The authors conclude that unanchored LTA, released into the mutant strain supernatant, competitively inhibits the effect of the wild-type strain's anchored LTA in promoting invasion. If so, one would predict that the wild-type supernatant, containing lower levels of soluble LTA than the mutant supernatant, would inhibit invasion less effectively. However, the wild-type supernatant actually enhanced BMEC invasion. These data again raise the question of whether there is a non-LTA structure anchored by the iagA gene product.

\section{Conclusions}

Overall, this work contributes significantly to our understanding of the molecular pathogenesis of invasive GBS infection. Specifically, the identification of the $i a g A$ gene product as a major contributor to GBS BMEC invasion in vitro and virulence in vivo is an exciting development. The finding that this gene product likely encodes a DGlcDAG synthase opens an avenue for inquiry into the role of such an enzyme in bacterial virulence.

These data invite inquiry in other areas as well: Do mutations in additional genes that contribute to LTA synthesis/anchoring/regulation influence the invasion phenotype? Does purified LTA or an LTAspecific antibody block invasion? What features distinguish meningitis-associated organisms/strains from those that express iagA and LTA but do not cause meningitis? Finally, does the iagA gene product act in conjunction with other recognized GBS invasins (e.g., $\beta$-hemoly$\sin$ (cytolysin) and, if so, how? Improved understanding of the molecular basis for the interactions of GBS with host epithelial and endothelial cells can be expected to enhance our ability to predict, diagnose, and prevent invasive GBS disease and our understanding of the pathogenic mechanisms of other organisms.

\section{Acknowledgments}

M.J. Baron and D.L. Kasper are supported by NIH grants AI59495 and N01-AI-25495, respectively.

Address correspondence to: Dennis L. Kasper, Harvard Medical School, Department of Medicine, 181 Longwood Avenue, Channing Laboratory, Boston, Massachusetts 02115, USA. Phone: (617) 432-3636 or (617) 525-2280; Fax: (617) 432-3639; E-mail: dennis_kasper@hms.harvard.edu.

1. Cywes, C., and Wessels, M.R. 2001. Group A Streptococcus tissue invasion by CD44-mediated cell signalling. Nature. 414:648-652.

2. Tamura, G.S., Kuypers, J.M., Smith, S., Raff, H., and Rubens, C.E. 1994. Adherence of group B streptococci to cultured epithelial cells: roles of environmental factors and bacterial surface components. Infect. Immun. 62:2450-2458.

3. Teti, G., Tomasello, F., Chiofalo, M.S., Orefici, G., and Mastroeni, P. 1987. Adherence of group B streptococci to adult and neonatal epithelial cells mediated by lipoteichoic acid. Infect. Immun. 55:3057-3064.

4. Bulgakova, T.N., Grabovskaya, K.B., Ryc, M., and Jelinkova, J. 1986. The adhesin structures involved in the adherence of group B streptococci to human vaginal cells. Folia Microbiol. (Praha). 31:394-401.

5. Bolduc, G.R., Baron, M.J., Gravekamp, C., Lachenauer, C.S., and Madoff, L.C. 2002. The alpha $\mathrm{C}$ protein mediates internalization of group B Streptococcus within human cervical epithelial cells. Cell. Microbiol. 4:751-758.

6. Baron, M., Bolduc, G., Goldberg, M., Auperin, T., and Madoff, L. 2004. Alpha C protein of group B Streptococcus binds host cell surface glycosaminoglycan and enters cells by an actin-dependent mechanism. J. Biol. Chem. 279:24714-24723.

7. Doran, K.S., Chang, J.C., Benoit, V.M., Eckmann, L., and Nizet, V. 2002. Group B streptococcal betahemolysin/cytolysin promotes invasion of human lung epithelial cells and the release of interleukin-8. J. Infect. Dis. 185:196-203.

8. Adderson, E., et al. 2003. Subtractive hybridization identifies a novel predicted protein mediat- ing epithelial cell invasion by virulent serotype III group B Streptococcus agalactiae. Infect. Immun. 71:6857-6863.

9. Schubert, A., et al. 2004. The fibrinogen receptor FbsA promotes adherence of Streptococcus agalactiae to human epithelial cells. Infect. Immun. 72:6197-6205.

10. Gutekunst, H., Eikmanns, B., and Reinscheid, D. 2004. The novel fibrinogen-binding protein FbsB promotes Streptococcus agalactiae invasion into epithelial cells. Infect. Immun. 72:3495-3504.

11. Cheng, Q., et al. 2002. Immunization with C5a peptidase or peptidase-type III polysaccharide conjugate vaccines enhances clearance of group B Streptococci from lungs of infected mice. Infect. Immun. 70:6409-6415.

12. Marques, M.B., Kasper, D.L., Pangburn, M.K., and Wessels, M.R. 1992. Prevention of C3 deposition by capsular polysaccharide is a virulence mechanism of type III group B streptococci. Infect. Immun. 60:3986-3993.

13. Edwards, M.S., Nicholson, W.A., Baker, C.J., and Kasper, D.L. 1980. The role of specific antibody in alternative complement pathway-mediated opsonophagocytosis of type III, group B Streptococcus. J. Exp. Med. 151:1275-1287.

14. Betz, A., and Goldstein, G. 1986. Specialized properties and solute transport in brain capillaries. Annu. Rev. Physiol. 48:241-250.

15. Doran, K.S., et al. 2005. Blood-brain barrier invasion by group B Streptococcus depends upon proper cell-surface anchoring of lipoteichoic acid. J. Clin. Invest. 115:2499-2507. doi:10.1172/JCI23829.

16. Ring, A., Weiser, J., and Tuomanen, E. 1998. Pneumococcal trafficking across the blood-brain barrier. Molecular analysis of a novel bidirectional pathway. J. Clin. Invest. 102:347-360.

17. Zaleznik, D., et al. 2000. Invasive disease due to group B Streptococcus in pregnant women and neonates from diverse population groups. Clin. Infect. Dis. 30:276-281.

18. Lindblom, G., Brentel, I., Sjolund, M., Wikander, G., and Wieslander, A. 1986. Phase equilibria of membrane lipids from Acholeplasma laidlawii: importance of a single lipid forming nonlamellar phases. Biochemistry. 25:7502-7510.

19. Neuhaus, F.C., and Baddiley, J. 2003. A continuum of anionic charge: structures and functions of D-alanyl-teichoic acids in gram-positive bacteria [review]. Microbiol. Mol. Biol. Rev. 67:686-723.

20. Granlund, E.M., Sellin, M., Holm, A., and Hakansson, S. 1993. Adherence and surface properties of buoyant density subpopulations of group B streptococci, type III. APMIS. 101:141-148.

21. Schwandner, R., Dziarski, R., Wesche, H., Rothe, M., and Kirschning, C. 1999. Peptidoglycanand lipoteichoic acid-induced cell activation is mediated by toll-like receptor 2. J. Biol. Chem. 274:17406-17409.

22. Kimpe, S.D., Kengatharan, M., Thiemermann, C., and Vane, J. 1995. The cell wall components peptidoglycan and lipoteichoic acid from Staphylococcus aureus act in synergy to cause shock and multiple organ failure. Proc. Natl. Acad. Sci. U. S. A. 92:10359-10363. 